\title{
Demonstration of Time Reversal Methods in a Multi-path Environment
}

\author{
K. Sarabandi* Fellow, IEEE, I. Koh Member, IEEE, and M. D. Casciato Member, IEEE \\ The University of Michigan \\ 1301 Beal Avenue \\ Ann Arbor, MI, 48109-2122 \\ Phone:(734) 936-1575, Email: saraband@eecs.umich.edu
}

\section{INTRODUCTION}

Time reversal methods offer a unique opportunity for solving the problem of electromagnetic (EM) wave propagation and focusing in a spatially varying (inhomogeneous) medium. While the concept of time reversal or applying time reversal mirrors to focus waves in spatially varying media is new to the field of EM wave propagation, it has been applied in the area of acoustics and ultrasonics for several years [1]. The basic premise is quite simple. Let an impulse source (in time and space) be transmitted into some general inhomogeneous medium, and consider the tangential surface fields determined on a closed surface surrounding the impulse. It can be shown mathematically that if these surface fields are conjugated and re-radiated in time reversed sequence, that the incoming wave, generated by the surface fields is identical to the outgoing wave, and the returning energy focuses on the original source point. In the frequency domain, this is equivalent to simply taking the complex conjugate of the received field, and reradiating it. This can be shown to be an application of reciprocity, which can be viewed as a spatio-temporal matched filter, similar to temporal matched filters cormmonly applied in radar and communications.

In practical applications of TRM, a source transmits through an inhomogeneous medium, to an a finite array. In the frequency domain, it can be shown through reciprocity that the following relationship holds:

$$
\mathbf{J}_{1} \cdot \mathbf{E}_{2}=\mathbf{J}_{2} \cdot \mathbf{E}_{1}
$$

where $\mathbf{E}_{1}, \mathbf{E}_{2}$ are electric fields caused by current sources $\mathbf{J}_{1}, \mathbf{J}_{2}$, respectively. Let $\mathbf{E}_{1}$ represents the fields caused by source $\mathbf{J}_{1}$, at the location of an array element, and $\mathbf{E}_{2}$ represent the fields caused by reradiation of this element, at the location of the original source $\mathbf{J}_{1}$. If the reradiated fields from the array element are caused by current source $\mathbf{J}_{2}$, and $\mathbf{J}_{2}=\mathbf{E}_{1}^{*}$, then the right side, and therefore the left side of (1) becomes purely real, and the received fields from the array element at the location of the original source thus are also purely real. Applying this to all elements in the array, the coherent sum of their fields at the original source point will add constructively, and focus the received energy at this point.

In any finite size array that occupies a limited spatial area the system is diffraction limited, however in acoustics, it has been shown that in an inhomogeneous medium a time reversal array is not always diffraction limited and can achieve super-resolution [2]. Basically scatterers near the transmitting array and/or focal point act as an extension of the array in the focusing process. In this paper the same phenomenon is shown to exist for EM waves.

II. Applications of TRM in Communications and Imaging

The application of time reversal methods to EM wave propagation offers the opportunity for a revolutionary change in currently applied methods in the transmission and focusing of signals through spatially varying or inhomogeneous media. Mathematically, time reversal 
methods allow for the determination of the system transfer function, or dyadic Green's function for any unknown media. An inhomogeneous spatial varying medium creates complex problems for both the communications system designer, as well as the radar engineer.

The significant multi-path inherent in such a propagation environment, can cause significant fading and delay spreads in a wireless communications system. Increasing the bandwidth of the system allows the receiver to discriminate between multi-path, however the total energy available from each energy path at the receiver is significantly diminished.

Imaging and identification of hard targets is usually achieved by applying high resolution synthetic aperture radar (SAR). When a target is embedded in a highly scattering, inhomogeneous medium, such as foliage or vegetation, however, it is not clear how to account for the defocusing effects of the medium and how to separate the clutter return from that of the target. Low frequency SAR is often used, as the inhomogeneities tend to be more transparent at higher wavelengths. However high resolution, low frequency systems require ultrawide bandwidth, are cumbersome to implement, and their image quality suffers from radio, TV, and other communications systems interference. Initial analysis of time reversal methods shows that they could significantly enhance the performance of radar imaging and detection of targets, and thus improve the performance of high resolution radar systems operating at higher frequencies.

III. Demonstration of TRM Focusing in a Forest Using a Physics-Based Fractal TREE MODEL

In order to demonstrate the feasibility of applying TRM to practical problems, a forest simulation tool, developed at the University of Michigan's Radiation Laboratory is applied to represent a highly scattering, inhomogeneous environment. The forest model is based on a statistical Lindenmayer fractal tree system and has been significantly validated. It is highly accurate, and includes extremely fine details of the propagation environment (for example, details of tree stands in a forest, including their structure, geometry, and orientation, as well as leaves, needles, tree trunks, and each individual branch of a tree) [3]-[5].

The physical scenario for this simulation is shown in Figure la, where a linear array, say of a SAR, is illuminating a forest. The objective is to demonstrate that the forest has the ability to defocus an array designed to focus in free space and to show that if the channel is known, focusing with super resolution is possible, using TRM methods. Polarimetric channel characterization is accomplished by placing a source inside a forest stand and calculating the radiated field (amplitude, phase, polarization) at the location of all array elements. TRM is then applied to this array distribution and re-radiated, and the complex field distribution excited by the array observed inside the forest, on a line perpendicular to the array, which passes through the focal point.

For this simulation 30 red pine trees are generated using the stochastic fractal model, and placed randomly around the focal point (location of source inside the forest), with a density of $0.05 \mathrm{~T} / \mathrm{m}^{2}$ (Figure $1 b$, focal point is at $x=0, y=0$, in the figure). The mean height of the trees, and the mean diameter of the tree trunks, are $15 \mathrm{~m}$ and $22 \mathrm{~cm}$, respectively. The moisture content of the tree needles is set at $0.62 \mathrm{~g} / \mathrm{cm}^{3}$, with that of the branches and tree trunks set to $0.42 \mathrm{~g} / \mathrm{cm}^{3}$. Note that each pine tree contains over 200,000 needles, and 1,800 branches. The ground plane is considered slightly rough, having a volumetric soil moisture content of $m_{\mathrm{v}}=0.18$ which consists of $50 \%$ sand and $10 \%$ clay. At this frequency the soil dielectric constant is calculated to be $\varepsilon_{g}=9.75-j 1.65$. The RMS height of the rough ground plane is fixed at $1 \mathrm{~cm}$, and the frequency is set at $3 \mathrm{GHz}$ The center of the antenna array (real aperture or synthetic) is located at a distance $R_{0}=14 \mathrm{~km}$ from the focal point, parallel to the $x$-axis, with a look angle of $45^{\circ}$ from nadir The array consists of 23 elements, spanning an aperture length of $1 \mathrm{~km}$ (see coordinate system in Figure 1a). In the field calculations, near-field and far-field scattering from all needles, branches, tree trunks, and their interaction with the ground plane are accounted for in a coherent fashion. Without loss of generality, each antenna is assumed to be an infinitesimal z-directed (vertical) dipole. 


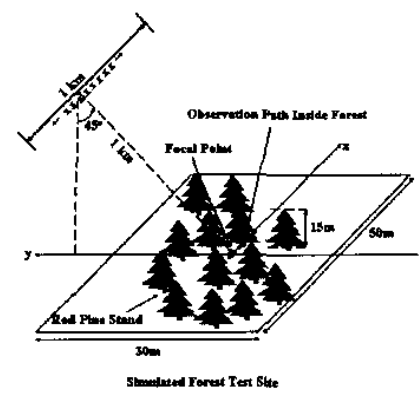

(a) Simulated forest scenario for TRM focusing demonstration

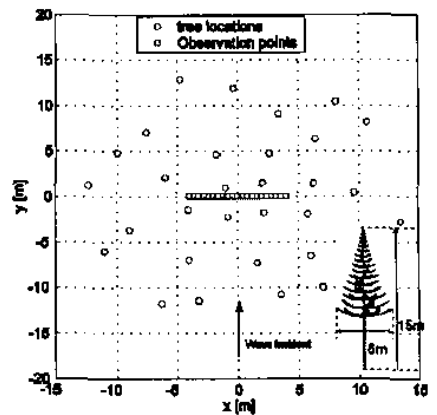

(b) Distribution of trees in a red pine stand and observation line (horizontal bar in figure, $y=0,-4<x<4)$.

Fig. 1. Simulated forest scenario and tree distribution for a red pine stand

In order to compare the capabilities of TRM to standard imaging techniques, such as $\mathrm{SAR}$, results are generated, which use the array discussed, to focus on a desired point in the forest, through both free space and the forest. This will demonstrate the defocusing effects of the forest, when applying standard array focusing techniques. To focus the array, in the standard fashion, a spherical phase and amplitude correction factor is applied along the array, and is given by $k_{0} x^{2} / R_{0}$ (note that this correction factor is symmetric about the array center, along the $x$ axis), where $x$ is the coordinate of the antenna element along the $\mathrm{x}$-axis, $R_{0}$ is distance from the array center, to the focal point $(14 \mathrm{~km})$, and $k_{0}$ is the free space propagation constant. Figure 2 shows the field distribution in the forest, along an observation line on the $x$-axis, parallel to the array. Observing first the field distributions using the standard array focusing, the defocusing effects of the highly scattering medium of the forest (curve "forest" in Figure 2), when compared to the free space case (in the absence of the forest, curve "Free-space" in Figure 2), are obvious. Now comparing these two cases with TRM focusing (curve "Focused", in Figure 2) the TRM pattern shows a significant improvement of $10 \mathrm{~dB}$, over the pattern using standard focusing, through the forest. What is of greater interest in the figure, is that TRM shows an improvement in focal width over the free space case, thus demonstrating that TRM can exceed the diffraction limit (superresolution). Critical in the application of TRM however, is to account for the polarimetric nature of the EM wave. The results in Figures $3 a$, and $b$ show the required magnitude and phase of both the $\mathrm{v}$ and $\mathrm{h}$ polarized array excitation to achieve focusing inside the forest. Remembering that TRM focusing is maximized by accounting for all the multi-path effects (i.e., TRM must maximize the energy received from all multi-path), including all field aspects (magnitude, phase, and polarization,), then if the TRM array is not polarization matched the focusing aspects of TRM cannot be fully exploited.

\section{Conclusion}

While this paper has demonstrated the feasibility of TRM to EM problems, future work must investigate and resolve many issues before TRM can be practically implemented in a communications or radar imaging system. These issues include, array size and spacing (for the TRM), the effects of the non-stationary behavior of the channel, and frequency/bandwidth. Relevant issues to be investigated for covert wireless communications include the tradeoff between spatial focusing and wideband transmission schemes, such 


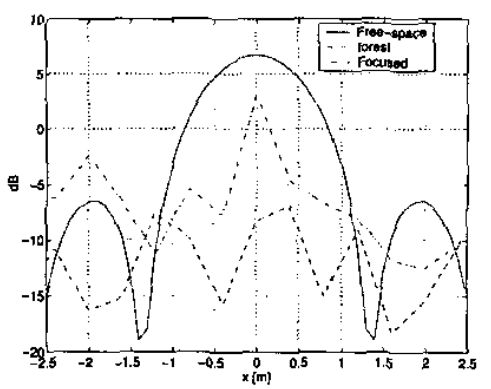

Fig. 2. Simulated array patterns for freespace (in the absence of the forest), inside the forest using freespace focusing ("forest" in figure legend), and focused under forest (using TRM, "Focused" in figure legend) Nate the super resolution feature of the focused pattern under the forest usiag TRM.

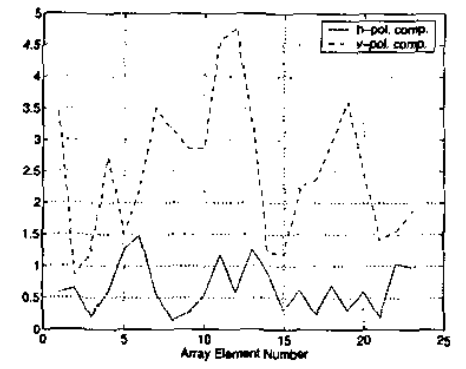

(a) Magnitude distribution of array excitation for both polarizations.

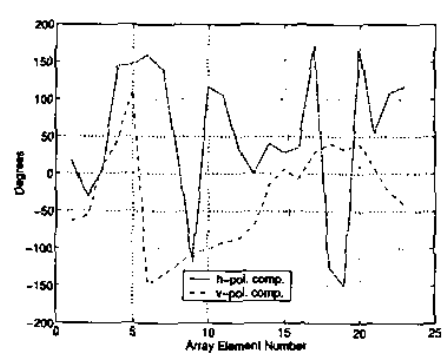

(b) Phase distribution of array excitation for both polarizations.

Fig. 3. Polarimetric complex feld distributions (magnitude and phase) on the array.

as ultrawideband, time-domain, and other spread spectrum techniques. Relevant issues to be investigated for radar target imaging and detection include the influence of various parameters on TRM focusing, including target parameters such as structure and electrical properties, channel parameters, including temporal variations and their effects on time reversal focusing, and system attributes, such as frequency, bandwidth, waveform, and polarization.

\section{REFERENCES}

[1] M. Fink and C. Prada, "Acoustic Time-Reversal Mirrors," Institute of Physics Publishing: Inverse Problems, vol. 17, pp. R1-R38, 2001.

[2] P. Blongren, G. Papanicolaou, and H. Zhao, "Super-resolution in Time-Reversal Acoustics," Submitted to the Journal of the Acoustical Society of Americe

3) I. Koh and K. Sarabandi, "Polarimetric Channel Characterization of Foliage for Performance Assessnent of GPS Receivers Under "Tree Canopies," IEEE Trans. Antenras Propagat., vol. 50, no. 5, pp. 713-726,

[4] K. Sarabandi, M. D. Casciato, and I. Koh, "Efficient Calculation of the Fields of a Dipole Radiating A bove 5. Y. C. L d ance Surface," JEEE Yrons. Antennas Propagat., vol. 50, no. 9, pp. 1222-1235, September 2002. Fractal-Generated Trees," IEEE Trons. on Geoscience and Remote Seneing, vol 37 no canopies using Jasuary 1999 